\section{Testing of Braden QD Scale for predicting pressure ulcer risk in the Pediatric Intensive Care Unit}

\author{
Jayanti Dwi Puspitasari1,2, \\ Nani Nurhaeni ${ }^{1}$, Fajar Tri Waluyanti1 \\ 1 Faculty of Nusing, Universitas \\ Indonesia; ${ }^{2}$ Dr. R. Soedjati Soemodiarjo \\ Hospital, Grobogan, Indonesia
}

\begin{abstract}
Children admitted to the Pediatric Intensive Care Unit (PICU) are at risk for pressure injury due to immobility and the challenge of positioning medical devices. However, a comprehensive instrument to assess pressure injury risk is still finite. This study aimed to test the validity and reliability of the Braden QD Scale. The design used was a Pearson correlation coefficients and Cronbach's alpha $(\alpha)$. Fifty-one pediatric patients below age 18 who had been bedridden for at least 24 hours were engaged through a consecutive sampling method. The results showed assessments made by using the Braden QD Scale revealed that $88.2 \%$ of patients were at risk for a pressure injury. The validity test results of the Braden QD Scale ranged between 0.532 and 0.833 . The reliability test results of the Braden QD Scale ranged between 0.756 and 0.834 . The sensitivity value is $100 \%$, specificity value is $40 \%$. In general, the study finding suggests that the Braden QD Scale is a valid and reliable instrument for predicting pressure injury risk. Nurses may employ this tool to assess pressure injury risk in pediatric patients.
\end{abstract}

\section{Introduction}

The Pediatric Intensive Care Unit (PICU) is a unit within a hospital that is equipped with staff and medical devices to provide care and treatment for critically ill patients. Critically ill patients require intensive care under which the patient is closely and continuously observed in order to identify physiological changes associated with the declining functions of body organs. ${ }^{1}$ Children admitted to the intensive care unit are at risk for pressure ulcers.

The prevalence of pressure injuries among hospitals is varied. A study by Schinder et al. reported that the incidence of pressure injury in children ranged between $3 \%$ and $10 \% .^{2}$ Kottner et al.'s study (2010) revealed that the prevalence of pressure ulcers in children who were admitted to the ward and PICU was $7 \%$ and $27 \%$, respectively. ${ }^{3}$ A study conducted by Rowe et al. (2018) in pediatric hospitals in the United States reported a surging incidence of Hospital Acquired Pressure Injury (HAPI) of up to $117 \%$ throughout 2013 and 2015, and the Pediatric Intensive Care Unit had the highest prevalence of HAPI. ${ }^{4}$ Curley et al. stated that 22 of 86 HAPI cases were associated with immobility and the rest were associated with medical devices. ${ }^{5}$ Widiawati et al. (2017) identified medical devices that were linked with pressure injury in the PICU of X hospital in Jakarta, including the endotracheal tube (ETT) in $13 \%$ of incidents, the orogastric tube (OGT) in 12\% of incidents, the nasogastric tube (NGT) in 11\% of incidents, and the oxygen saturation probe in $6 \%$ of incidents. ${ }^{6}$ It can be concluded that prolonged immobilization and the challenge of positioning medical devices are major causes of pressure injury in the PICU.

Pressure injury causes physical discomfort that may affect a patient's health status or even lead to death. The management and treatment of a pressure injury require a longer time, which prolongs the hospital stay and increases the cost of care. ${ }^{7}$ Alderden et al. reported that HAPI may prolong patients' length of stay, increase morbidity, and aggravate their suffering. ${ }^{8}$ Preventive measures are critical in order to prevent the development of a pressure injury throughout hospitalization.

A one-week observation of medical device-related pressure injury in the PICU X hospital in Jakarta revealed that 3 of 7 observed patients were affected by pressure injury due to pulse oximetry and 1 of them was caused by the tensimeter cuff pressure that is always attached. All of them were affected by a grade 1 pressure injury. Interviews with nurses revealed barriers contributing to their neglect of preventive measures for pressure injury associated with medical devices. The first barrier was the number of priority interventions that they should perform. The second one was a lack of comprehension concerning pressure injury associated with medical devices in the intensive care unit. The third barrier was that pressure injury associated with medical devices was not included as a quality indicator of nursing service in the hospital. The fourth one was a lack of instruments to predict the pressure injury risk associated with medical devices. So, a validity and reliability test became the priority to obtain a valid and reliable tool.

\section{Materials and Methods}

The Pearson correlation product moment was applied to test the validity and
Correspondence: Nani Nurhaeni, Pediatric Nursing Department, Faculty of Nursing, Universitas Indonesia, Jalan Prof. Dr. Bahder Djohan, Kampus UI Depok, West Java, 16424 Indonesia.

Tel.: +6221.78849120 - Fax: +6221.7864124.

E-mail: nani-n@ui.ac.id

Key words: Braden QD scale; pressure injury risk; validity; reliability; PICU.

Acknowledgments: The writer expresses gratitude to all respondents and their families, the PICU nurses at Dr. Cipto Mangunkusumo Hospital Jakarta.

Funding: This study was supported by Hibah PITTA B 2019, funded by DRPM Universitas Indonesia (No. NKB-0496/UN2.R3.1/HKP.05. 00/2019).

Contributions: JDP designed research, conduct research, collect data, wrote the manuscript in consultation on with NN, FTW; NN, and FTW verified the analytical methods. All authors discussed the results and contributed to the final manuscript.

Ethical approval: Ethical clearance issued by the Research Ethics Committee of the Faculty of Nursing Universitas Indonesia (No. 10/UN2 F12.D/HKP.02.04/2019).

Conflict of interest: The authors declare that have no conflict of interest.

This work is licensed under a Creative Commons Attribution NonCommercial 4.0 License (CC BY-NC 4.0).

(C) Copyright: the Author(s), 2020

Licensee PAGEPress, Italy

Pediatric Reports 2020; 12(s1):8694

doi:10.4081/pr.2020.8694

reliability of the instrument. The sample size formula for a correlational study was applied and required 51 children for the research subject. The consecutive sampling method was employed. The inclusion criteria were children between age 1 and 18 who had been bedridden for at least 24 hours. The total participants in this research were 51 children. The research was conducted in the PICU within 14 weeks (January 1, 2019, to April 5, 2019). The instrument used in this research to collect data on the participant characteristics was an observation checklist that covered age, sex, and the Braden QD Scale tool. Ethical clearance was issued by the Ethical Committee of the Faculty of Nursing of Universitas Indonesia (No. 10/UN2.F12. D/HKP.02.04/2019). 


\section{Results}

Table 1 reveals that the majority of participants were boys (54.9\%). Most of them were aged between 1 month and 1 year or infanthood $(37.3 \%)$.

Table 2 indicates that $88.2 \%$ of pediatric patients were at risk for pressure injury based on an assessment conducted by using the Braden QD Scale. The assessment obtained by using the Braden Q Scale revealed that only $70.6 \%$ of the patients were at risk for pressure injury. The scoring of the pressure injury risk of each tool was based on the mean as the cut-off point, that is, 13 and 16 , respectively.

\section{Validity test}

The validity test of the Braden QD scale revealed the $\mathrm{r}$-value ranging between 0.532 and 0.833 and between 0.528 and 0.804 for the Braden Q scale with the $r$ table of 0.279 , which indicates the validity of the instrument.

\section{Reliability test}

Table 3 suggests that in general, both the Braden QD Scale and Braden Q Scale were reliable with Cronbach's alpha $(\alpha)>0.6$.

\section{Sensitivity and specificity test}

The sensitivity, specificity, and accuracy value can be calculated based on Table 4 . The calculation is performed as follows.

\section{Sensitivity}

The sensitivity value is a diagnostic test result that represents the capacity of a test to identify people with an illness of the entire population of people with illness. The calculation of the sensitivity value based on the table is $36 /(36+0) \times 100 \%$, which equals $100 \%$. The sensitivity value of $100 \%$ implies that $100 \%$ of the risk for impaired skin integrity in children admitted to the PICU can be identified with the Braden QD Scale.

\section{Specificity}

The specificity value is a diagnostic test result that represents the capacity of a test to identify people without an illness (negative test) the entire population of subjects without illness. The calculation of the specificity value based on the table is $6 /(6+9) \times 100 \%$, which equals $40 \%$.

\section{Accuracy}

The accuracy value is a comparison between a positive subject with an illness and a subject without the illness of all screened subjects. The accuracy of the Braden QD Scale in this diagnostic test is $82.4 \%((36+6) / 51 \times 100 \%)$ or good within a classification that includes the categories of very weak $(>50-60)$, weak $(>60-70)$, moderate $(>70-80)$, good $(>80-90)$, and very good ( $>90-100)$. The result suggests that if the Braden QD Scale was applied to assess pressure injury risk in children admitted to the PICU, the scale would result in a good conclusion in predicting the risk in 82 of 100 screened children.

\section{Discussion}

The results suggest that the majority of participants were infants (1 month-1 year old). Lorento et al.'s study revealed that children below one year of age had the highest prevalence of pressure sores. ${ }^{9}$ Age

Table 1. Distribution of participants based on gender and age in PICU.

\begin{tabular}{lcc} 
Variable & Frequency (n) & Pencentage (\%) \\
Gender & & \\
Boys & 38 & 45.9 \\
Girls & 23 & 45.1 \\
\hline Age & & \\
Infant (1 month-1 year) & 19 & 37.3 \\
Toddler (1-3 years) & 8 & 15.7 \\
\hline Pre-school (3-6 years) & 8 & 15.71 \\
School-age (6-12 years) & 7 & 3.7 \\
\hline Adolescence (12-18 years) & 9 & 17.6 \\
\hline
\end{tabular}

Table 2. Distribution of participants based on pressure injury by using Braden QD Scale and Braden Q Scale in PICU.

\begin{tabular}{lcccc} 
Instrument & Not at risk for pressure injury & At risk for pressure injury \\
& F & $\%$ & F & $\%$ \\
Braden QD & 6 & 11.8 & 45 & 88.2 \\
Braden Q & 15 & 29.4 & 36 & 70.6 \\
\hline
\end{tabular}

Table 3. The difference in reliability test results.

\begin{tabular}{lcccl} 
Parameter & $\begin{array}{c}\text { Palpha } \\
\text { Braden QD }\end{array}$ & $\begin{array}{c}\text { Palpha } \\
\text { Braden Q }\end{array}$ & Roritical & Criteria \\
Mobility & 0.790 & 0.728 & 0.6 & Reliable \\
Sensory perception & 0.756 & 0.724 & 0.6 & Reliable \\
\hline Friction and shear & 0.766 & 0.739 & 0.6 & Reliable \\
Nutrition & 0.815 & 0.754 & 0.6 & Reliable \\
\hline Tissue perfusion & 0.796 & 0.747 & 0.6 & Reliable \\
Number of medical devices & 0.834 & - & 0.6 & Reliable \\
\hline Repositionability & 0.781 & - & 0.6 & Reliable \\
Activity & - & 0.742 & 0.6 & Reliable \\
\hline Moisture & - & 0.724 & 0.6 & Reliable \\
\hline
\end{tabular}

Table 4. Sensitivity and specificity test of Braden QD Scale on the Braden Q Scale in PICU.

\begin{tabular}{llccc} 
Instrument & \multicolumn{2}{c}{ Braden Q } & Total \\
& & At risk for & Not at risk for \\
& pressure injury & pressure injury & \\
\multirow{2}{*}{ Braden QD } & At risk for pressure injury & 36 & 9 & 45 \\
& Not at risk for pressure injury & 0 & 6 & 6 \\
Total & & 36 & 15 & 51 \\
\hline
\end{tabular}


is a factor that contributes to pressure injury as the skin structure keeps changing within the first 18 years of life and its function will continue to develop. 10 Furthermore, a child's skin contains more water that is easier to evaporate and absorb than an adult's skin, which makes it vulnerable to damage. ${ }^{11}$ The second factor is that children would not yet be able to communicate the perceived discomfort, and the last factor is that they are inclined to be fussy or even to unplug or remove installed medical devices.

The results are supported by several studies addressing the impact of age on the development of pressure injury. Schindler $e t$ al. stated that age below 2 years was a risk factor for pressure ulcers. Habibalah and Tubaishat reported that pressure injury in the PICU mostly affected children under 1 year (72.1\%). ${ }^{12}$ A study by Schluer et al. revealed that pressure injury predominantly developed in patients who were admitted to the PICU (44\%) and neonatology ward (43\%). ${ }^{13}$

A hospital is required to develop a guide based on Evidence-Based Nursing $(E B N)$ in order to prevent HAPI. The standard procedure serves as a guide for nurses to implement uniformed intervention. According to Rowe et al., Nurse Driven Pathway (NDP) can be applied to prevent HAPI. NDP is proven to be effective in reducing HAPI by up to $57 \%$ in the PICU and improving nurses' compliance with the implementation of the pressure ulcer prevention bundle by up to $66 \%{ }^{4}$

The prevention of medical device-related pressure injury (MDPI) is a challenge for nurses, as a patient who is admitted to the intensive care unit requires medical devices and yet devices may also harm the patient when not properly monitored. ${ }^{2}$ According to Johson et al., preventive measures of MDPI included assessing the skin that is in contact with the medical device, repositioning the medical device, and proper placement of the medical device. An instance of proper placement of the medical device is inserting a nasogastric tube that is adjusted based on the patient's size, ensuring the tube is not too large or too small, and fixing it with tape for safety purposes. ${ }^{14}$

Based on the sensitivity and specificity value, the Braden QD Scale is able to $100 \%$ predict the risk for pressure injury with a specificity value of $40 \%$. The result disproves Curley et al.'s study, which revealed a sensitivity value of $86 \%$ and a specificity value of $59 \%$ at the 13 cut-off point. ${ }^{4}$ The higher sensitivity value is directly proportional to the increased score on the Braden QD Scale since this study was conducted on PICU patients who were mostly affected by immobility and underwent placements with more than 8 medical devices.
A study by Curley et al. reported that the sensitivity and specificity value of the Braden Q Scale were $88 \%$ and $58 \%$, respectively. Lu et al. indicated that the sensitivity and specificity value of the Braden Q Scale were 0.71 and 0.53 , respectively, with 19 as the cut-off point when it was applied in the PICU; the low sample size presumably contributed to the low sensitivity and specificity. ${ }^{15}$ Liao et al. reported that the sensitivity and specificity value of the Braden Q Scale were 0.73 and 0.61 , respectively, which implies a moderate sensitivity value and a low specificity value. Therefore, it is necessary to develop and modify the instrument that confirms the current situation and conditions for the best result. 11

There are several weaknesses in the Braden Q Scale, including an inability to identify medical device-related pressure injury, as the scale was originally applied for children aged 3 months to 8 years, and inapplicable for patients with congenital heart disease. ${ }^{16}$ Flaws within the Braden Q Scale prompted Quigley and Quartrano to modify and simplify it to the Braden QD Scale. The Braden QD Scale offers several advantages over the Braden Q Scale. The first advantage is that the Braden QD Scale is capable of predicting pressure injury risk due to immobility and the use of medical devices. Second, this scale was examined based on participants ranging from preterm age up to 21 years old and those with various medical conditions (cardiovascular, neurology, surgery, acute, and chronic diseases), thus making it easier to apply to the pediatric or adult population admitted into intensive care.

\section{Conclusions}

The Braden QD Scale is a valid and reliable tool that is applicable to assess pressure injury risk in children between infanthood and 18 years with immobility and medical devices.

\section{References}

1. Norton-Westwood D. The health-care environment through the eyes of childdoes it soothe or provoke anxiety? Int J Nurs Pract 2012;18:7-11.

2. Schindler CA, Mikhailov TA, Kuhn $\mathrm{EM}$, et al. Protecting fragile skin: Nursing interventions to decrease development of pressure ulcers in pediatric intensive care. Am J Crit Care 2011; 20:26-34.

3. Kottner J, Wilborn D, Dassen T. Frequency of pressure ulcers in the paediatric population: A literature review and new empirical data. Int J Nurs Stud 2010;47:1330-40.

4. Rowe AD, Mccarty K, Huett A. Implementation of a nurse driven pathway to reduce incidence of hospital acquired pressure injuries in the pediatric intensive care setting. J Pediatr Nurs 2018;41:104-9.

5. Curley MAQ, Hasbani NR, Quigley $\mathrm{SM}$, et al. Predicting pressure injury risk in pediatric patients: The Braden QD Scale. J Pediatr 2018;192:189-95.

6. Widiati E, Nurhaeni N, Gayatri D. Medical-device related pressure injury to children in the intensive care unit. Compr Child Adolesc Nurs 2017;40:69-77.

7. Tayyib N, Coyer F, Lewis P. A two-arm cluster randomized control trial to determine the effectiveness of a pressure ulcer prevention bundle for critically ill patients. J Nurs Scholarsh 2015;3:237-47.

8. Alderden J, Rondinelli J, Pepper G, et al. Risk factors for pressure injuries among critical care patients: A systematic review. Int J Nurs Stud 2017;71:97-114.

9. Lorente SMM, Sánchez SE, Molina GP, et al. Prevalence of pressure ulcers in the paediatric population and in primary health care: An epidemiological study conducted in Spain. J Tissue Viability 2018;27:221-5.

10. Blume UP, Hauser M, Stamatas GN, et al. Skincare practices for newborns and infant: Review of the clinical evidence for best practices. Pediatr Dermatol 2012;29:1-14.

11. Liao Y, Gao G, Mo L. Predictive accuracy of the Braden Q Scale in risk assessment for paediatric pressure ulcer: A meta-analysis. Int J Nurs Sci 2018;5:419-26.

12. Habiballah L, Tubaishat A. The prevalence of pressure ulcers in the paediatric population. J Tissue Viability 2016;25: 127-34

13. Schluer AB, Schols JM, Halfens RJ. Pressure ulcer treatment in pediatric patients. Adv Skin Wound Care 2013;26:504-10.

14. Johnson M, Barnett C, Wand T, White K. Medical device-related pressure injuries: An exploratory descriptive study in an acute tertiary hospital in Australia. J Tissue Viability 2017;26:246-53.

15. Lu YF, Yang Y, Wang Y, et al. Predicting pressure ulcer risk with the Braden Q Scale in Chinese pediatric patients in ICU. Chin Nurs Res 2015;2:1-5.

16. Chamblee TB, Pasek AC, Caillouette $\mathrm{CN}$, et al. Continuing education: How to predict pediatric pressure injury risk with the Braden QD Scale. Am J Nurs. 2018;118:34-43. 